\title{
Ciclo de Mejora en la docencia de Gestión de Enfermería, mediante el trabajo en equipo y colaborativo
}

\author{
MARÍA DOLORES GUERRA-MARTÍN \\ Universidad de Sevilla \\ Departamento de Enfermería \\ guema@us.es \\ ORCID: https://orcid.org/0000-0002-1409-1287 \\ D.O.I.: http://dx.doi.org/10.12795/JDU.2018.i01.93 \\ Pp.: 1657-1675
}

\section{Resumen}

Se presenta un Ciclo de Mejora Docente llevado a cabo en la asignatura de Ética y gestión de Enfermería, con una duración de 30 horas. El ciclo se ha realizado en un grupo grande y se ha abordado la parte de gestión de enfermería. Se han trabajado doce problemas interrelacionados en tres unidades temáticas (UT): Sistemas sanitarios (UT 1) y Proceso administrativo en sus dos fases de preparación (UT 2) y de ejecución (UT 3). Los estudiantes han participado de manera colaborativa en pequeños equipos, realizando búsquedas de información autónomas y creando preguntas que incluían en la aplicación Kahoot y que después respondían todos los que asistían a clase. Se han realizado cuestionarios de conocimientos iniciales y finales en cada UT, comprobando el avance en la escalera de aprendizaje de los estudiantes. 
Palabras clave: Ética y Gestión de Enfermería, Grado en Enfermería, Docencia Universitaria, Experimentación Docente Universitaria.

\section{Breve descripción del contexto}

El Ciclo de Mejora Docente (CMD) se ha llevado a cabo en la asignatura de Ética y Gestión de Enfermería de $3^{\text {er }}$ curso del Grado en Enfermería de la Universidad de Sevilla (US). Esta asignatura es obligatoria y está planificada en el primer cuatrimestre. Tiene seis créditos ECTS, de los cuales, cuatro se imparten en grupo grande (tres para Gestión de enfermería y uno de Ética enfermera).

El CMD se ha realizado con el grupo 1 con 51 matriculados, en la parte de Gestión de Enfermería, la cual se ha impartido desde el 24/10/18 hasta el 20/11/18. El CMD ha tenido una duración de 30 horas. En relación a la asistencia ha sido irregular, desde los primeros días con aproximadamente 40 estudiantes, hasta los últimos días de clase con 15-20. Decir que esta disminución en el número de estudiantes ha sido debida a que tuvieron un examen de otra asignatura el día 19/11/18.

La participación de los asistentes ha sido muy alta, ya que han trabajado en equipos de manera colaborativa, realizando lecturas expuestas en la enseñanza virtual, además, de búsquedas de información autónomas y la elaboración y contestación de preguntas mediante la aplicación Kahoot. 


\section{Diseño previo del Ciclo de Mejora Docente}

Modelo metodológico consolidado.

Hasta ahora no había llevado a cabo ningún CMD en la docencia en grupo grande. Mi modelo habitual se correspondía con una clase expositiva en la cual intentaba fomentar la participación de los estudiantes mediante preguntas, y los contenidos y materiales docentes estaban a disposición de los estudiantes mediante la enseñanza virtual (EV).

En este curso he cambiado hacia un modelo que he ido consolidando en la docencia en grupo pequeño (seminarios). En este sentido, iniciaba la clase haciendo un esbozo o síntesis de la materia a tratar y/o de lo impartido en la clase anterior, después se repartían los estudiantes por equipos pequeños, y trabajaban de una manera colaborativa (Tourón y Santiago, 2015) para conseguir un aprendizaje autodirigido o self-directed learning (Marcelo, 2010), mediante lecturas del material docente expuesto en la EV, después planteaban sus dudas o problemas que hubieran encontrado tras las lecturas de los textos y realizaban búsquedas autónomas de información para intentar resolverlas (Guerra-Martín et al., 2013; Guerra-Martín et al., 2014).

Después, si aún tenían dudas o discrepancias, las iba tratando con cada equipo o en conjunto con toda la clase, si estas eran generalizadas. Posteriormente, los estudiantes pasaban a elaborar preguntas tipo test (similares a las del examen) y sus contestaciones, y las iban introduciendo en la plataforma Kahoot, previo visto bueno de la profesora.

Una vez finalizado el trabajo en equipo, terminaba la clase con una sintesis y conclusiones de lo tratado, para acabar recordándoles el tema de la siguiente clase, animándoles a que se lo leyeran fuera del aula, además, les insistía en que prestaran atención a distintas noticias que pudieran estar relacionadas con la temática, las cuales, se comentaban después en las clases. 
Como sintesis, decir que la manera de afrontar la tarea de enseñar durante este curso, se ha correspondido con el modelo basado en la reelaboración de las ideas de los estudiantes (De Alba y Porlán, 2017), el cual represento en la figura 1.

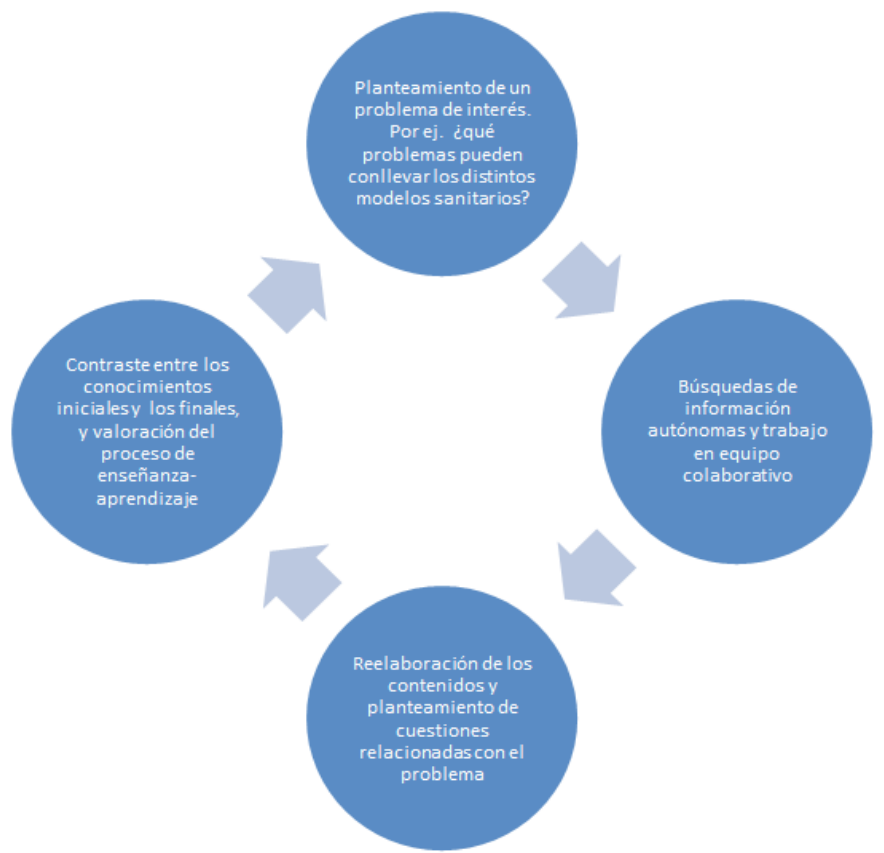

Figura 1. Representación de mi modelo metodológico. Basado en De Alba y Porlán, 2017.

\section{Mapas de contenidos y problemas.}

Los contenidos de la asignatura los agrupé en tres Unidades Temáticas (UT): Sistemas Sanitarios; Fase de Preparación del Proceso Administrativo: Planificación y Organización y Fase de Ejecución del Proceso Administrativo: Dirección y Calidad. En las figura 2 se puede observar el mapa de contenidos de UT sobre de los Sistemas Sanitarios y en la figura 3 del Proceso Administrativo, con la fase de Preparación y la de Ejecución. (Los mapas de contenidos y problemas: otra manera de diseñar la enseñanza. Formadores: José Antonio Pineda Alfonso y Ana Belén Feria Bourrellier. Curso organizado por el ICE. Universidad de Sevilla). 


\section{SISTEMAS SANITARIOS}

Sauitarios-Éticos-Sociales-Económicos-Políticos-Legales

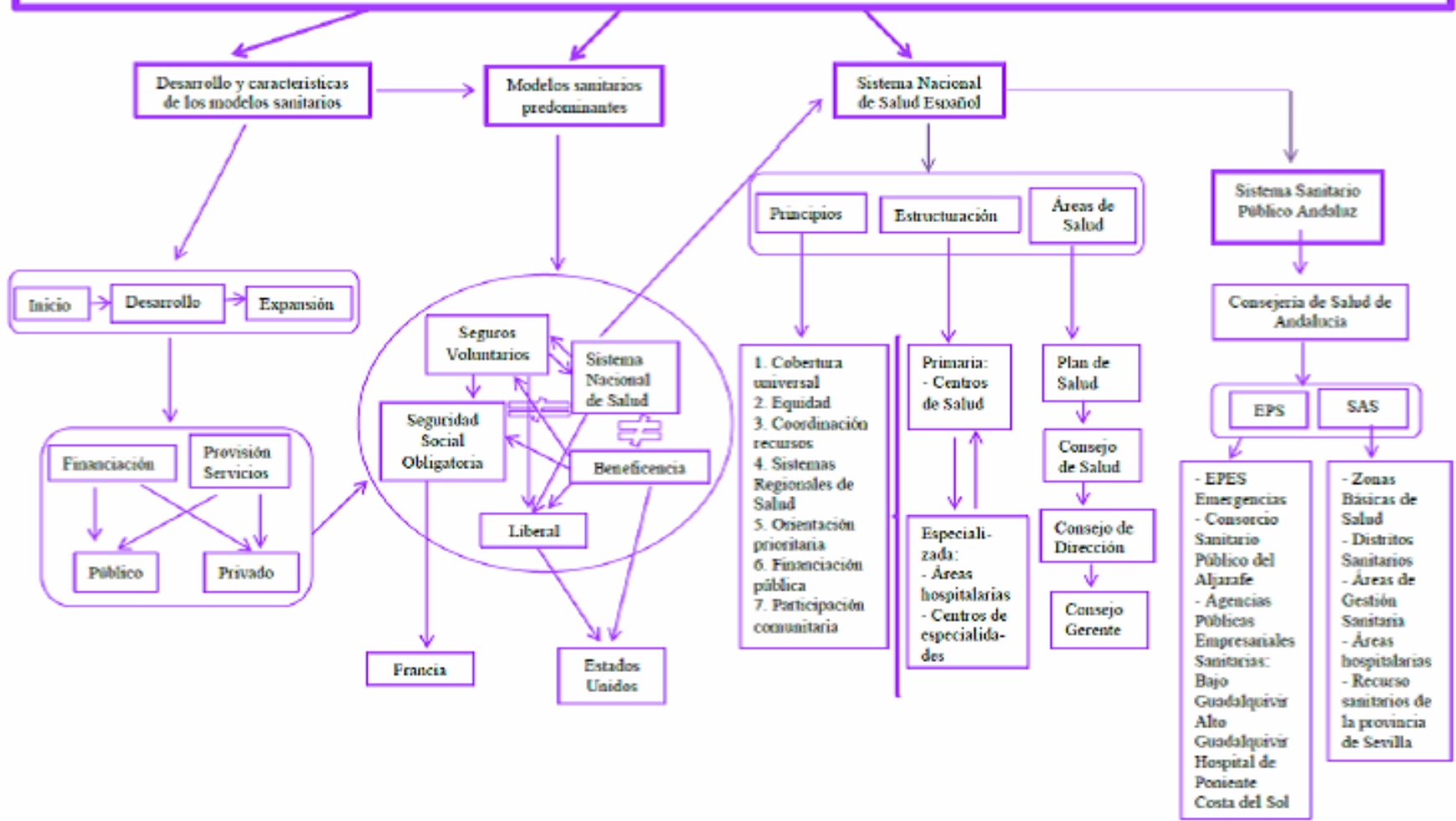

Figura 2. Mapa de contenidos sobre los Sistemas Sanitarios.

Jornadas de Formación e Innovación Docente del Profesorado | № 1 (2018)

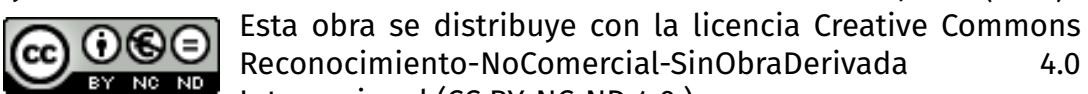
Internacional (CC BY-NC-ND 4.0.) 


\section{PROCESO ADMINISTRATIVO}

¿Cuál es el método de resolución de problemas en la gestión sanitaria? ¿Qué etapas se siguen?

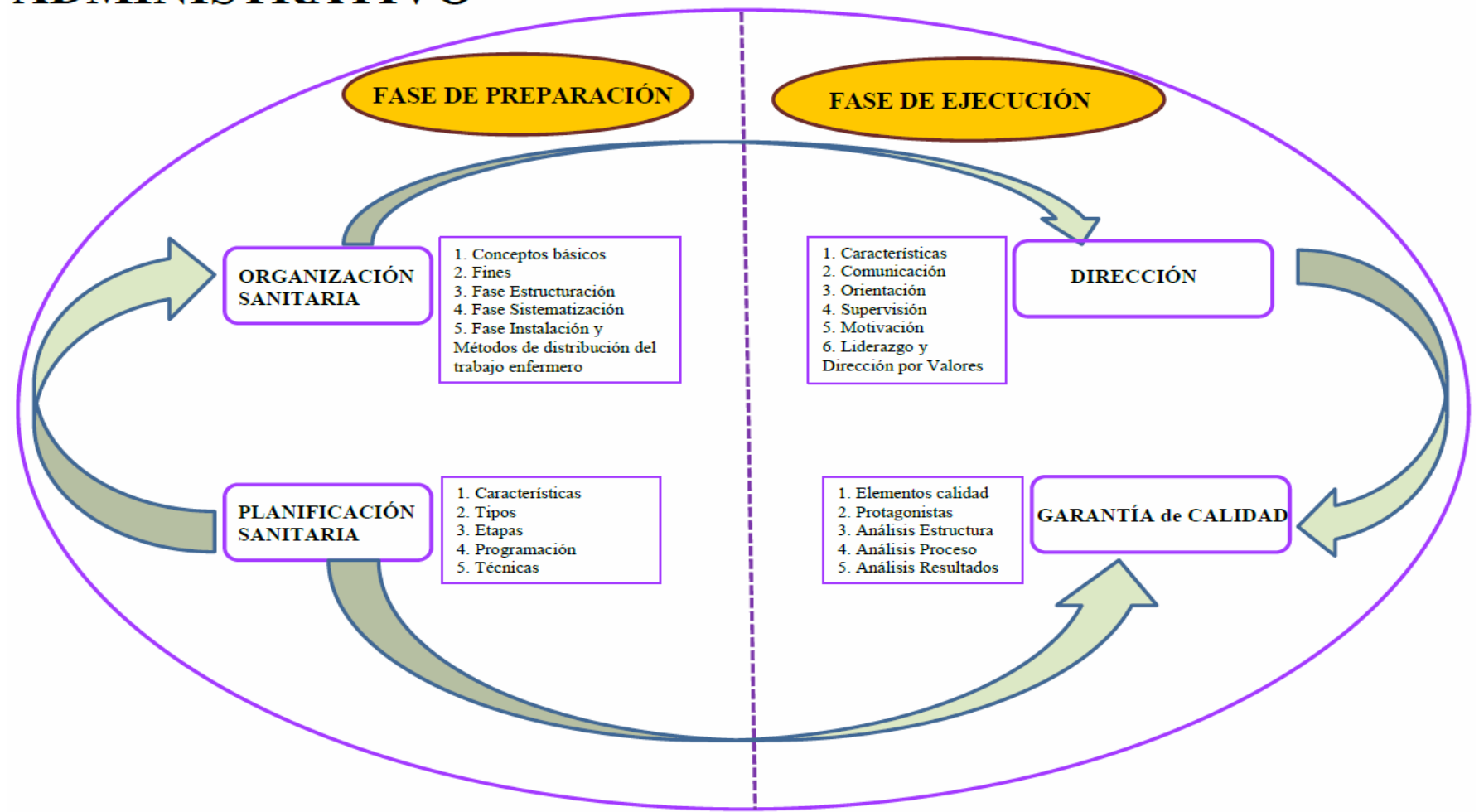

Figura 3. Mapa de contenidos sobre el Proceso Administrativo.

Jornadas de Formación e Innovación Docente del Profesorado | № 1 (2018)

(c) (i) $\ominus$ Esta obra se distribuye con la licencia Creative Commons 
En los mapas de contenidos expuestos en el apartado anterior, están las relaciones entre los distintos contenidos de la asignatura. A continuación se presenta el temario de la asignatura, dividido en tres UT y los contenidos conceptuales incluidos en cada una de ellas.

UT 1: Sistemas Sanitarios. Contenidos conceptuales:

1. Antecedentes de los Sistemas Sanitarios y Modelos Sanitarios.

2. Sistemas de Salud de EEUU, Francia y Reino Unido.

3. Sistema Nacional de Salud Español.

4. Sistema Sanitario Público de Andalucía.

UT 2: Proceso Administrativo: Planificación Sanitaria y Organización de los Servicios Sanitarios. Contenidos conceptuales:

5. Planificación Sanitaria y técnicas de Planificación.

6. Organización Sanitaria: Estructuración, Sistematización e Instalación.

7. Métodos de Distribución del Trabajo Enfermero.

UT 3: Proceso Administrativo: Dirección y Garantía de Calidad de los Servicios Sanitarios.

8. Dirección y Motivación.

9. Liderazgo y Dirección por Valores.

10. Garantía de Calidad de los Servicios.

\section{Cuestionario inicial y final para los estudiantes y secuencia de actividades.}

Para la evaluación de conocimientos he utilizado el gestor de encuestas OPINA, mediante el cual he ido elaborando cuestionarios iniciales y finales de conocimientos de cada una de las tres UT. Las preguntas tenían cada una cinco opciones de respuesta: 1. Nada. 2. Casi nada. 3. Algo. 4. Bastante. 5. Mucho. En la tabla 1 se exponen las actividades llevadas a cabo con su temporalización y las preguntas realizadas en cada UT. 
Secuencia de actividades y temporalización.

\section{Fechas/horas/Actividades desarrolladas}

24/11 (2 h). Presentación. Proyecto Docente: Contenidos, metodología y evaluación. Fomento de la participación. Planteamiento de dudas. Solicitud de expectativas e inquietudes con la asignatura. Debate. Cuestionario INICIAL de conocimientos (UT 1: Sistemas Sanitarios):

1. ¿Qué características tienen los Modelos Sanitarios: Liberal, Seguros Voluntarios y Seguros Sociales Obligatorios? 2. ¿Qué características tienen Modelos Sanitarios: Asistencia Pública de Beneficencia y Sistema Nacional de Salud? 3. ¿Cuáles son los principios que rigen la Estructura y Funcionamiento del Sistema Nacional de Salud Español? 4. ¿Cuántas Agencias Públicas Empresariales Sanitarias hay en Andalucía y cuáles son las finalidades de cada una de ellas?

Planteamiento del Problema: ¿Qué problemas socioeconómicos, sanitarios y éticos se plantearon durante los Antecedentes y desarrollo de los Sistemas Sanitarios?

25/11 (1 h). Planteamiento del Problema: ¿Qué problemas socioeconómicos, sanitarios y éticos se plantean en los Sistemas Sanitarios, según sea la Financiación y Provisión de servicios, pública y/o privada?

01/10 (2 h). Planteamiento del Problema: ¿Qué problemas socioeconómicos, sanitarios y éticos se plantean en los distintos Modelos Sanitarios? Nuevo planteamiento del Problema: ¿Cuáles son las diferencias socioeconómicas, sanitarias y éticas entre el Sistema Nacional de Salud Español y el anterior modelo de Seguridad Social Obligatoria?

02/10 (1 h). Planteamiento del Problema: ¿Cómo influyen las Áreas de Salud en el ámbito socioeconómico, ético, sanitario en España?

08-10 (2 h). Planteamiento del Problema: ¿Qué acciones llevan a cabo los Organismos No sanitarios adscritos a la Consejería de Salud para solventar los problemas socioeconómicos, sanitarios y éticos en Andalucía?

Nuevo planteamiento del Problema: ¿Qué acciones llevan a cabo los Organismos sanitarios (Agencias Públicas Empresariales Sanitarias) adscritos a la Consejería de Salud para solventar los problemas socioeconómicos, sanitarios y éticos en Andalucía?

09/10 (1 h). Planteamiento del Problema: ¿Qué acciones se llevan a cabo desde Atención Primaria y desde Atención Hospitalaria para solventar los problemas socioeconómicos, sanitarios y éticos en Andalucía? 
15/10 (2 h). Cuestionario FINAL de conocimientos (UT 1: Sistemas Sanitarios).

Cuestionario INICIAL de conocimientos sobre la fase de Preparación del Proceso Administrativo (UT 2).

Presentación de la UT 2: Fase de Preparación del Proceso Administrativo. Cuestionario INICIAL de conocimientos sobre la fase de Preparación del Proceso Administrativo: 1 ¿Qué características tiene la Planificación Sanitaria? 2. ¿Cuáles son las Técnicas de Planificación Sanitaria? 3. ¿Qué es la Organización Sanitaria y cuáles son sus fases? 4. ¿Cuáles son los Sistemas de Asignación de Cuidados Enfermeros?

Planteamiento del Problema: ¿Cómo influyen las características de la Planificación en la Gestión de Servicios Sanitarios?

16/10 (1 h). Planteamiento del Problema: ¿Para qué sirven las Técnicas de Planificación Sanitaria en la Gestión de Servicios?

22/10 (2 h). Planteamiento del Problema: ¿Cómo influyen las Características de la Organización Sanitaria en la Gestión de Servicios?

Nuevo Planteamiento del Problema: ¿Cuáles son las Fases de la Organización Sanitaria y que trabajo conllevan dentro de la Gestión Sanitaria?

23/10 (1 h). Planteamiento del Problema: ¿Qué características tiene la Estructuración y Sistematización en la Organización Sanitaria?

29/10 (2 h). Planteamiento del Problema: ¿Qué características tiene la Instalación en la Organización Sanitaria y cómo influencian las políticas de contratación de personal?

Nuevo planteamiento del Problema: ¿Cómo se realiza el sistema de Clasificación de pacientes y el cálculo de plantilla de enfermería?

30/10 (1 h). Planteamiento del Problema: ¿Cuáles son los Sistemas de Asignación de Cuidados Enfermeros en Atención Primaria y en Atención Hospitalaria?

05/11 (2 h). Cuestionario FINAL de conocimientos sobre la fase de Preparación del Proceso Administrativo (UT 2).

Cuestionario INICIAL de conocimientos sobre la fase de Ejecución del Proceso Administrativo (UT 3):

1. ¿Qué elementos son importantes a tener en cuenta dentro de la etapa de la Dirección Sanitaria? 2. ¿Cuáles son los factores motivadores según Herzberg y qué dice la teoría Y de McGregor? 3. ¿Cuáles son los estilos de liderazgo según la autoridad del líder y para qué sirve la Dirección por Valores? 4. ¿Cuáles son los problemas y las estrategias de mejora de la Calidad Asistencial en el Proceso según Donabedian?

Planteamiento del Problema: ¿Cuáles son los Elementos que integran la Dirección Sanitaria? 
06/11 (2 h). Planteamiento del Problema: ¿Qué características tienen la Información y Comunicación dentro de la Dirección Sanitaria?

Nuevo planteamiento del Problema: ¿Qué características tienen la Orientación y la Supervisión dentro de la Dirección Sanitaria?

12/11(2 h). Planteamiento del Problema: ¿Qué es la Motivación y cómo influye en el trabajo?

Nuevo planteamiento del Problema: ¿Cuáles son las Teorías sobre la Motivación laboral?

13/11(2 h). Planteamiento del Problema: ¿Qué es el Liderazgo y cuáles son los Estilos de Liderazgo?

Planteamiento del Problema: ¿Cuáles son las Teorías sobre el Liderazgo?

19/11(2 h). Planteamiento del Problema: ¿Cuál ha sido la Evolución en las formas de dirigir las empresas?

Nuevo planteamiento del Problema: ¿Qué es la Dirección por Valores y que finalidades cumple en las empresas?

20/11(2 h). Planteamiento del Problema: ¿Cuáles son los elementos y protagonistas de la Calidad Asistencial y como se investiga y garantiza la calidad?

Realización de los cuestionarios:

- Cuestionario FINAL de conocimientos sobre la fase de Ejecución del Proceso Administrativo (UT 3)

- Cuestionario de opinión y satisfacción con el proceso de enseñanza-aprendizaje

Finalización del CMD, informando a los estudiantes que se les proporcionará información, una vez analizados los datos del proceso y de los resultados.

\section{Aplicación del Ciclo de Mejora Docente}

\section{Relato resumido de las sesiones.}

En la tabla 2 se han expuesto las actividades realizadas en el CMD. Para complementarla, hay que hacer referencia a que, al empezar cada nueva clase, se hizo un recordatorio del tema a tratar y una sintesis de la clase anterior. Así mismo, al empezar cada UT se hizo una presentación de los contenidos de la misma. 
Por otro lado, tras el planteamiento del problema en todas las clases se hizo lo siguiente: Trabajo en equipos de 3-5 estudiantes. Planteamientos de dudas y/o problemas: Lecturas colgadas EV y búsquedas de información. Resolución de problemas y dudas. Elaboración e introducción de preguntas tipo test en Kahoot y contestaciones. Sintesis y conclusiones de los temas trabajados. Recordatorio del tema de la siguiente clase y del trabajo fuera del aula. Por último, decir que tras la realización de los cuestionarios finales de cada UT se hizo una puesta en común.

\section{Evaluación del aprendizaje de los estudiantes.}

\section{Valoración previa sobre las inquietudes y expectativas.}

El primer día de clase les hice una pregunta abierta a los estudiantes sobre las inquietudes y expectativas que tenían con la asignatura, a esta pregunta contestaron 38 estudiantes. A continuación se exponen algunas de las contestaciones literales que hicieron:

"Aprender la organización y funcionamiento de nuestro sistema de salud, así como el papel de la enfermera"

"En mi opinión, es una asignatura novedosa de la que creo que no conozco apenas. Por lo tanto, espero adquirir todos los conocimientos necesarios sobre ella"

"Aprender sobre la gestión de los hospitales y Atención Primaria, además de la ética enfermera y de las leyes que rigen la práctica enfermera"

"Me intriga la forma en la que vamos a trabajar estos temas, que son muy desconocidos para mí"

"Pensaba que sería bioética, hacia la que tenía altas expectativas porque pienso que es muy interesante, pero acabo de descubrir que no, así que sinceramente siento curiosidad por aprender un poco más de qué va la asignatura porque parece ser que hasta ahora estaba equivocada" 
"Mis expectativas serían conocer el campo legal de mi profesión, los aspectos que me conciernen. Que se considera calidad asistencial y si nuestra asistencia cumple dichos requisitos. Además de conocer la documentación que tengo que manejar en mí trabajo"

"Me parece que es una asignatura interesante ya que creo que es importante que si trabajamos en el ámbito sanitario conozcamos los distintos modelos que existen, como funcionan..."

"Espero conocer los distintos métodos de organización de la sanidad ya que de todo el ámbito sanitario es lo que más desconozco"

"Es una asignatura que a priori me interesa pues aun estando ya en tercero de enfermería tengo un gran desconocimiento con lo relacionado al sistema de salud andaluz"

"Aprender la organización del sistema sanitario español y todo lo relacionado con ello, incluido aspectos de interés como responsabilidad enfermera o consentimientos informados"

"Creo que es una asignatura que me permitirá conocer y delimitar las competencias de enfermería y cómo manejar las responsabilidades legales"

\section{Escalera de aprendizaje.}

En la tabla 3 se detallan los resultados obtenidos por los estudiantes en la escalera de aprendizaje de cinco niveles que se describen a continuación: Nivel 1: No escribe nada o la contestación no es concordante con lo consultado. Nivel 2: Escribe muy pocas ideas que apenas concuerdan con lo consultado. Nivel 3: Escribe algunas ideas demostrando un conocimiento suficiente sobre la materia. Nivel 4: Escribe bastantes ideas demostrando bastante conocimiento sobre la materia. Nivel 5: Escribe muchas ideas demostrando mucho conocimiento sobre la materia. En general, se observa un aumento en todos los niveles de aprendizaje en los cuestionarios finales de conocimientos. 
Tabla 3

Niveles de aprendizaje obtenidos por los estudiantes en el CMD.

\begin{tabular}{|c|c|c|c|c|c|c|c|c|c|c|}
\hline \multirow{2}{*}{ Preguntas } & \multicolumn{2}{|c|}{ Nivel 1 } & \multicolumn{2}{c|}{ Nivel 2 } & \multicolumn{2}{c|}{ Nivel 3 } & \multicolumn{2}{c|}{ Nivel 4 } & \multicolumn{2}{c|}{ Nivel 5 } \\
\cline { 2 - 10 } & Pre & Post & Pre & Post & Pre & Post & Pre & Post & Pre & Post \\
\hline 1 & $47,7 \%$ & $1,3 \%$ & $42,0 \%$ & $3,8 \%$ & $10,2 \%$ & $45,0 \%$ & $0,0 \%$ & $46,3 \%$ & $0,0 \%$ & $3,8 \%$ \\
\hline 2 & $42,0 \%$ & $2,5 \%$ & $38,6 \%$ & $5,0 \%$ & $19,3 \%$ & $45,0 \%$ & $0,0 \%$ & $42,5 \%$ & $0,0 \%$ & $5,0 \%$ \\
\hline 3 & $47,7 \%$ & $2,5 \%$ & $42,0 \%$ & $2,5 \%$ & $10,2 \%$ & $48,8 \%$ & $0,0 \%$ & $40,0 \%$ & $0,0 \%$ & $6,3 \%$ \\
\hline 4 & $76,1 \%$ & $7,5 \%$ & $20,5 \%$ & $13,8 \%$ & $3,4 \%$ & $36,3 \%$ & $0,0 \%$ & $38,8 \%$ & $0,0 \%$ & $3,8 \%$ \\
\hline 5 & $51,7 \%$ & $0,0 \%$ & $34,5 \%$ & $5,3 \%$ & $13,8 \%$ & $42,1 \%$ & $0,0 \%$ & $47,4 \%$ & $0,0 \%$ & $5,3 \%$ \\
\hline 6 & $72,4 \%$ & $0,0 \%$ & $24,1 \%$ & $5,3 \%$ & $3,4 \%$ & $31,6 \%$ & $0,0 \%$ & $63,2 \%$ & $0,0 \%$ & $0,0 \%$ \\
\hline 7 & $75,9 \%$ & $0,0 \%$ & $17,2 \%$ & $5,3 \%$ & $6,9 \%$ & $47,4 \%$ & $0,0 \%$ & $42,1 \%$ & $0,0 \%$ & $5,3 \%$ \\
\hline 8 & $86,2 \%$ & $5,3 \%$ & $6,9 \%$ & $5,3 \%$ & $6,9 \%$ & $31,6 \%$ & $0,0 \%$ & $52,6 \%$ & $0,0 \%$ & $5,3 \%$ \\
\hline 9 & $55,6 \%$ & $0,0 \%$ & $33,3 \%$ & $0,0 \%$ & $11,1 \%$ & $0,0 \%$ & $0,0 \%$ & $87,5 \%$ & $0,0 \%$ & $12,5 \%$ \\
\hline 10 & $33,3 \%$ & $0,0 \%$ & $38,9 \%$ & $0,0 \%$ & $27,8 \%$ & $0,0 \%$ & $0,0 \%$ & $87,5 \%$ & $0,0 \%$ & $12,5 \%$ \\
\hline 11 & $5,6 \%$ & $0,0 \%$ & $38,9 \%$ & $0,0 \%$ & $50,0 \%$ & $0,0 \%$ & $5,6 \%$ & $81,3 \%$ & $0,0 \%$ & $18,8 \%$ \\
\hline 12 & $77,8 \%$ & $0,0 \%$ & $16,7 \%$ & $0,0 \%$ & $5,6 \%$ & $0,0 \%$ & $0,0 \%$ & $93,8 \%$ & $0,0 \%$ & $6,3 \%$ \\
\hline
\end{tabular}

\section{Evaluación de las percepciones de la evolución del aprendizaje.}

En este apartado se incluyen las contestaciones que han realizado los estudiantes, en los cuestionarios iniciales y finales de conocimientos, sobre sus percepciones de los conocimientos. Se realizaron 12 preguntas cerradas (cuatro por cada UT) con cinco opciones de respuesta: 1: Nada. 2: Casi nada. 3. Algo. 4. Bastante. 5. Mucho. En la tabla 4 se exponen los resultados, resaltando un mayor aumento de conocimientos, según afirmaciones de los estudiantes, en la UT 3 sobre la Dirección y Calidad de los servicios sanitarios. Sobre la pregunta no 11 hay que decir que sale algo más elevada en los conocimientos previos porque ese tema se acababa de impartir la parte la relacionada con el liderazgo, de una manera bastante práctica, en la docencia en los seminarios (grupo pequeño). 
Tabla 4

Conocimientos iniciales y finales desde el punto de vista de los estudiantes.

\begin{tabular}{|l|c|c|}
\hline Cuestiones sobre conocimientos & $\begin{array}{c}\text { Cuestionario Inicial } \\
\text { (pretest) }\end{array}$ & $\begin{array}{c}\text { Cuestionario final } \\
\text { (postest) }\end{array}$ \\
\hline $\begin{array}{l}\text { 1. Sobre los modelos sanitarios: } \\
\text { liberal, seguros voluntarios y se- } \\
\text { guros sociales obligatorios }\end{array}$ & $1,63 \pm 0,67$ & $3,48 \pm 0,70$ \\
\hline $\begin{array}{l}\text { 2. Sobre los modelos sanitarios: } \\
\text { asistencia pública de beneficen- } \\
\text { cia y sistema nacional de salud }\end{array}$ & $1,77 \pm 0,75$ & $3,43 \pm 0,78$ \\
\hline $\begin{array}{l}\text { 3. Sobre los principios que rigen } \\
\text { la estructura y funcionamiento } \\
\text { del sistema nacional de salud } \\
\text { español }\end{array}$ & $1,63 \pm 0,67$ & $3,45 \pm 0,76$ \\
\hline $\begin{array}{l}\text { 4. Sobre las agencias públi- } \\
\text { cas empresariales sanitarias de } \\
\text { Andalucía }\end{array}$ & $1,27 \pm 0,52$ & $3,18 \pm 0,98$ \\
\hline $\begin{array}{l}\text { 5. Sobre las características de la } \\
\text { planificación sanitaria }\end{array}$ & $1,62 \pm 0,73$ & $3,06 \pm 0,25$ \\
\hline $\begin{array}{l}\text { 6. Sobre las técnicas de planifica- } \\
\text { ción sanitaria }\end{array}$ & $1,31 \pm 0,54$ & $3,53 \pm 0,70$ \\
\hline $\begin{array}{l}\text { 7. Sobre la organización sanitaria } \\
\text { y sus fases }\end{array}$ & $1,31 \pm 0,60$ & $3,58 \pm 0,61$ \\
\hline $\begin{array}{l}\text { 8. Sobre los sistemas de asigna- } \\
\text { ción de cuidados enfermeros }\end{array}$ & $1,21 \pm 0,60$ & $3,47 \pm 0,70$ \\
\hline $\begin{array}{l}\text { 9. Sobre las etapas de la direc- } \\
\text { ción sanitaria }\end{array}$ & $1,56 \pm 0,71$ & $3,13 \pm 0,34$ \\
\hline $\begin{array}{l}\text { 10. Sobre los factores motiva- } \\
\text { dores según Herzberg y sobre la } \\
\text { teoría y de Mcgregor? }\end{array}$ & $1,94 \pm 0,80$ \\
\hline $\begin{array}{l}\text { 11. Sobre los estilos de liderazgo } \\
\text { según la autoridad del líder y so- } \\
\text { bre la dirección por valores }\end{array}$ & $2,56 \pm 0,71$ & \\
\hline $\begin{array}{l}\text { 12. Sobre los problemas y las es- } \\
\text { trategias de mejora de la calidad } \\
\text { asistencial en el proceso según } \\
\text { Donabedian }\end{array}$ & $1,28 \pm 0,58$ & \\
\hline
\end{tabular}

Jornadas de Formación e Innovación Docente del Profesorado I № 1 (2018) Esta obra se distribuye con la licencia Creative Commons Reconocimiento-NoComercial-SinObraDerivada Internacional (CC BY-NC-ND 4.0.) 


\section{Opiniones y satisfacción de los estudiantes con el CMD.}

En la tabla 5 se presentan los resultados del cuestionario de satisfacción de los estudiantes con el proceso de enseñanza-aprendizaje llevado a cabo en el CMD.

\section{Tabla 5.}

Satisfacción de los estudiantes.

\begin{tabular}{|l|c|c|}
\hline \multicolumn{1}{|c|}{$\begin{array}{c}\text { Preguntas sobre la valoración de la } \\
\text { satisfacción }\end{array}$} & Media & Desviación típica \\
\hline $\begin{array}{l}\text { Unidad temática sobre los sistemas sanita- } \\
\text { rios (UT 1) }\end{array}$ & 4,23 & 0,439 \\
\hline $\begin{array}{l}\text { Unidad temática sobre la planificación y la } \\
\text { organización (UT 2) }\end{array}$ & 4,23 & 0,439 \\
\hline $\begin{array}{l}\text { Unidad temática sobre la dirección y la ca- } \\
\text { lidad (UT 3) }\end{array}$ & 4,31 & 0,480 \\
\hline $\begin{array}{l}\text { En general, cuál ha sido su satisfacción de } \\
\text { las tres UT }\end{array}$ & 4,38 & 0,506 \\
\hline
\end{tabular}

En el cuestionario se les preguntó también a los estudiantes, sobre los aspectos que más les habían gustado del CMD y sobre los que se podían mejorar. A continuación se exponen algunos de los comentarios literales de los estudiantes sobre los aspectos que más le gustaron:

"El bloque de dirección y calidad"

"Lo que más me ha gustado ha sido la forma dinámica de impartir las clases, siempre con un buen ambiente entre la profesora y los alumnos"

"Trabajo en equipo"

"Es dinámico"

"La realización de preguntas relacionadas con los temas creo que nos sirve para enfatizar los puntos más importantes"

"Lo que más me ha gustado ha sido el dinamismo de la clase, en consecuencia está no me ha resultado pesada" 
"Me ha parecido muy útil el proceso de aprendizaje y me ha servido para facilitarme el aprendizaje"

"Hacer preguntas parecidas a las que van a ser en el examen"

"El poder participar nosotros mismos y generar preguntas que nos hagan entenderlas mejor nosotros mismos"

"Las clases se hacen muy dinámicas con el Kahoot"

"Me ha gustado la idea del Kahoot porque te hace leerte cada semana los temas e ir pensando en posibles preguntas de manera práctica"

"Me gusta que sea una clase dinámica, que hagamos cuestionarios y trabajemos en equipo"

A continuación se exponen los comentarios literales de los estudiantes sobre los aspectos a mejorar:

"Volver a la clase magistrales"

"El temario de organización, dividirlo en más temas porque si no se hace muy largo"

"Alternar clases normales con grupales"

"Dar una explicación previa más extensa de los temas y después, la realización del kahoot"

"Creo que las clases serían más productivas si en vez de solo leer los temas y hacer las preguntas, se profundizara antes con una explicación de las unidades, al menos para darnos cuenta de los puntos más importantes"

"Yo cambiaría el orden del proceso, daría una exposición del tema y luego seguiría con la dinámica participativa"

\section{Evaluación del Ciclo de Mejora Docente}

\section{Aspectos de la experiencia que se pretenden incorporar a toda la práctica docente habitual.}

Decir que voy a continuar en los siguientes cursos con las dinámicas seguidas este curso en las clases, aunque, tendré en cuenta las opiniones de los estudiantes y llevaré 
a cabo una mayor profundización al inicio de los temas y antes de que estos se pongan a trabajar en equipo.

El trabajar mediante problemas y preguntas de investigación significa un reto para los estudiantes, este desafío les motiva más a los estudiantes que la mera clase expositiva, en la cual se intentaba fomentar la participación sin hacerles partícipes de su proceso de enseñanza-aprendizaje y sin hacerles responsables del mismo.

\section{Principios didácticos argumentados que han guiado la experiencia y que deben permanecer en el futuro.}

Para que los estudiantes consigan las habilidades y destrezas necesarias para la gestión en enfermería, se ha fomentado la enseñanza activa mediante el manejo de bases de datos específicas de ciencias de la salud y la adaptación de los contenidos al mercado laboral (Guerra-Martín, 2014), por ejemplo, realizando un cálculo de plantilla de enfermería o llevando a cabo una técnica de planificación sanitaria.

Se han integrado los nuevos conocimientos con los saberes previos para lograr su aplicación en diferentes contextos (Julián, 2010; Guerra-Martín et al., 2014), como pueden ser la gestión de los distintos servicios hospitalarios o de los centros de salud, por ejemplo, mediante el conocimiento de los departamentos o servicios y de los organigramas de las distintas instituciones. De esta manera los estudiantes han conseguido mejor la comprensión de los conocimientos, a través de sus experiencias y reflexiones, conectando con sus redes de aprendizaje, pudiendo así tomar sus propias decisiones fundamentadas (Siemens, 2004). Para ello, se han conectado los conocimientos con las prácticas clínicas.

Se ha fomentado también, un clima que ha propiciado el interés y la motivación en los estudiantes, favoreciendo la responsabilidad y la toma de decisiones de estos en 
su proceso de aprendizaje. Además, se ha conseguido una participación activa y una actitud proactiva. A la vez que, se han promovido las búsquedas de información autónomas (Guerra-Martín et al., 2013; Guerra-Martín et al., 2014) y se ha potenciado el estudio fuera del aula.

La importancia de adquirir la competencia de las búsquedas autónomas de información durante la carrera, es porque este aprendizaje les va a servir a los estudiantes no solo durante su periodo de vida universitaria, sino también, posteriormente a lo largo de su vida profesional (Guerra-Martín et al., 2013; Guerra-Martín et al., 2014).

También, se han tenido en cuenta, los intereses y necesidades de las personas implicadas en la formación (Amador, 2001; Guerra-Martín et al., 2014), mediante la atención personalizada a los estudiantes por parte del profesorado, y el compromiso ético de una gestión basada en valores compartidos entre los estudiantes y profesores, como la confianza, la creatividad y la honestidad, entre otros (García y Dolan, 2003), sin perder de vista la mejora del rendimiento profesional, pero garantizando siempre la calidad asistencial.

Jornadas de Formación e Innovación Docente del Profesorado | № 1 (2018) Esta obra se distribuye con la licencia Creative Commons 


\section{Referencias bibliográficas}

Amador, L., Monreal, M.C. y Marco, M.J. (2001). El adulto: etapas y consideraciones para el aprendizaje. Eúphoros, 3, 97-112.

De Alba, N. y Porlán, R. (2017). La metodología de enseñanza. En R. Porlán (Coord.), Enseñanza universitaria. Cómo mejorarla (37-53). Madrid: Ediciones Morata.

Fernández-Gámez, D. y Guerra-Martín, M. D. (2016). Aprendizaje inverso en formación profesional: opiniones de los estudiantes. International Journal of Technology and Educational Innovation, Innoeduca 2 (1): 29-37.

García, S. y Dolan, S. (2003). La Dirección por Valores. Madrid: ESADE.

García, E., Porlán, R. y Navarro, E. (2017). Los fines y los contenidos. En R. Porlán (Coord.), Enseñanza universitaria. Cómo mejorarla (55-72). Madrid: Ediciones Morata.

Guerra-Martín M. D., Lima-Serrano, M., Zambrano-Domínguez, E. M. y Fernández-Rodríguez, V. (2013). Evaluación de una intervención sobre busquedas de información científica para estudiantes de enfermería. Texto \& Contexto- Enfermagem, 22 (3): 619-628. doi: http://dx.doi. org/10.1590/S0104-07072013000300007

Guerra-Martín MD. (2014). Tutoring as a way of achieving employability for nursing students at the University of Seville. Procedia-Social and Behavioral Sciences, 139: 479-486. Higher Education: The challenge of employability (XIII International Congress of Educational Theory). doi: $10.1016 /$ j.sbspro.2014.08.049

Guerra-Martín, M. D, Lima-Serrano, M., Zambrano-Domínguez, E. M. y Lima-Rodríguez, J. S. (2014). ¿Es efectivo el aprendizaje sobre alfabetización en información para estudiantes de enfermería? Enfermería Global, 13 (4): 90-102. doi: http://dx.doi.org/10.6018/eglobal.13.4.182541

Julián, J. F. y Narváez, C.G. (2010). Motivación y Ejercicio Docente en la Educación Superior: Una Aproximación Conceptual. Educere, 48, 21-27.

Marcelo, C. (2010). Autoformación para el siglo XXI. En J. Gairín ('Coord.). Nuevas estrategias formativas por las organizaciones (pp.141-170). Madrid: Wolters.

Siemens, G. (2004). Conectivismo: una teoría de aprendizaje para la era digital. http://d.scribd.com/docs/1yhhhthpoaervbohwzkc.pdf

Tourón, J. y Santiago, R. (2015). El modelo Flipped Learningy el desarrollo deltalento en la escuela. Revista de Educación, 368: 196-231. doi:10.4438/1988-592X-RE-2015-368-288 